\title{
Evaluation of carbapenem-resistant Enterobacteriaceae in a tertiary-level reference hospital in Rio Grande do Sul, Brazil
}

\author{
Vinícius Victor Lorenzoni ${ }^{[1],[2], ~ D a n i e l l y ~ d a ~ C o s t a ~ S i l v a ~}{ }^{[1],[2], ~ R o b e r t a ~ F i l i p i n i ~ R a m p e l o t t o ~}{ }^{[1],[2],}$ \\ Patrícia Chaves Brites ${ }^{[3]}$, Bárbara Villa ${ }^{[2],[3]}$ and Rosmari Hörner ${ }^{[1]}$
}

[1]. Departamento de Análises Clínicas, Universidade Federal de Santa Maria, Santa Maria, RS, Brasil. [2]. Programa de Pós-Graduação em Ciências Farmacêuticas, Universidade Federal de Santa Maria, Santa Maria, RS, Brasil. [3]. Laboratório de Biologia Molecular, Hospital Universitário de Santa Maria, Santa Maria, RS, Brasil.

\begin{abstract}
Introduction: The rapid global spread of carbapenem-resistant Enterobacteriaceae (CRE) is a threat to the health system. Methods: We evaluated the antimicrobial susceptibility profiles of $70 \mathrm{CRE}$ isolated in a tertiary hospital in Brazil between August and December 2015, and determined their resistance mechanisms. Results: The most prevalent microorganism was Klebsiella pneumoniae (95.7\%); it showed high-level resistance to carbapenems ( $>98 \%)$, with sensitivity to colistin $(91.4 \%)$ and amikacin (98.6\%). The $b l a_{\mathrm{KPC}}$ gene was detected in $80 \%$ of the CRE isolates. Conclusions: Evaluation of bacterial resistance contributes to an appropriate treatment, and the reduction of morbimortality and dissemination of resistance.
\end{abstract}

Keywords: Enterobacteriaceae. Klebsiella pneumoniae carbapenemase. Multiresistance.

Bacterial resistance is a serious health problem, leading to relapsing nosocomial infections, reduced drug efficacy, and expensive and imprecise treatment, and resulting in high morbidity and mortality rates ${ }^{1}$. The spread of carbapenemresistant Enterobacteriaceae (CRE) is threatening the health system, since the bacteria show multiple resistance mechanisms, such as the production of carbapenemases [e.g., Klebsiella pneumoniae carbapenemase (KPC); metallo- $\beta$-lactamases (MBL); and oxacillinases (OXA)] and efflux pumps, and porin $\operatorname{loss}^{2}$.

Since the spread of CRE is a worldwide problem, studies focusing on the characterization of resistance mechanisms and on the understanding of epidemiology at infection sites are required to inform an adequate empirical therapy and control the dissemination of resistance ${ }^{3}$. Therefore, the aim of the present study was to evaluate the antimicrobial susceptibility profiles of 70 CRE isolates obtained between August and December 2015 at the Hospital Universitário de Santa Maria (HUSM) (Santa Maria, Rio Grande do Sul, Brazil) and to determine their resistance mechanisms. The isolates were obtained from several clinical specimens and identified using the Vitek ${ }^{\circledR}$ automated system (bioMérieux, Marcy-l'Étoile, France).

Antimicrobial resistance of the isolates was evaluated against antimicrobial agents described in Table 1. The minimum

Corresponding author: Dra. Rosmari Hörner.

e-mail: rosmari.ufsm@gmail.com

Received 19 May 2017

Accepted 30 June 2017 inhibitory concentration (MIC) values were determined using the Advanced Expert System (AES) program in Vitek ${ }^{\circledR}$; these values were interpreted according to the criteria established by the Clinical and Laboratory Standards Institute (CLSI) and the technical standard no. 01/2013 from the Agência Nacional de Vigilância Sanitária (ANVISA) ${ }^{4,5}$. Phenotypic methods, such as disc diffusion tests with enzymatic inhibitors [phenylboronic acid (PBA); ethylenediaminetetraacetic acid (EDTA)], biochemical tests Carba NP [Carbapenemase NordmannPoirel $(\mathrm{CN})]$ and Blue-Carba (BC), and molecular methods [polymerase chain reaction (PCR)] were used to evaluate the resistance mechanisms. The disk diffusion method identifies KPC-producing strains using carbapenem disks supplemented with PBA; it identifies MBL-producing strains using the chelating agent EDTA ${ }^{4}$. The $\mathrm{CN}$ biochemical test is based on the hydrolysis of the $\beta$-lactam ring of imipenem. The strains were considered to be carbapenemase producers when a color change of a solution containing the phenol red indicator was observed. The $\mathrm{BC}$ test is based on the same principle as $\mathrm{CN}$, except that the bromothymol blue indicator is used ${ }^{6}$. To confirm the production of carbapenemases, PCR was performed to detect the presence of the $b l a_{\mathrm{KPC}}, b l a_{\mathrm{OXA}-48}, b l a_{\mathrm{NDM}}$, and $b l a_{\mathrm{GES}}$ genes ${ }^{7}$. The sensitivity and specificity of the different phenotypic methods were calculated considering PCR as the standard methodology ${ }^{6}$.

During the study period, 70 isolates were obtained from patients infected or colonized by CRE, being that each isolate corresponded to only one clinical specimen per patient. $K$. pneumoniae was the prevailing microorganism (67 isolates; 95.7\% of all CRE isolates), followed by Escherichia coli (1;1.4\%), Citrobacter freundii $(1 ; 1.4 \%)$, and Enterobacter cloacae (1; 
TABLE 1

Antimicrobial susceptibility profile of 70 CRE isolated between August and December 2015 at the HUSM, Santa Maria/RS, Brazil.

\begin{tabular}{|c|c|c|c|}
\hline Antimicrobial agents & MIC range $(\mu \mathrm{g} / \mathrm{mL})$ & $\operatorname{MIC50}(\mu \mathrm{g} / \mathrm{mL})^{*}$ & Resistant n (\%) \\
\hline Ampicillin & $1-32$ & $\geq 32$ & $70(100.0)$ \\
\hline Cefepime & $1-64$ & $\geq 64$ & $68(97.1)$ \\
\hline Ceftazidime & $1-64$ & $\geq 64$ & $69(98.6)$ \\
\hline Ciprofloxacin & $0.25-16$ & $\geq 4$ & $66(94.3)$ \\
\hline Colistin & $0.5-16$ & $\leq 0.5$ & $6(8.6)$ \\
\hline Ertapenem & $0.5-8$ & $\geq 8$ & $70(100.0)$ \\
\hline Gentamicin & $1-16$ & $\geq 16$ & $46(65.7)$ \\
\hline Piperacillin-tazobactam & $4-128$ & $\geq 128$ & $69(98.6)$ \\
\hline Tigecycline & $0.25-8$ & $\geq 8$ & $66(94.3)$ \\
\hline
\end{tabular}

CRE: carbapenem-resistant Enterobacteriaceae; HUSM: Hospital Universitário de Santa Maria; MIC: minimum inhibitory concentration. *MIC50, concentration required to inhibit the growth of $50 \%$ of microorganism.

$1.4 \%$ ). The specimen type from which the CRE were isolated most frequently was the rectal swab used in the epidemiological surveillance research $(32 ; 45.7 \%$ of 70 clinical specimens), followed by the urine $(21 ; 30 \%)$, sputum $(3 ; 4.3 \%)$, feces $(3 ; 4.3 \%)$, blood $(2 ; 2.9 \%)$, sacral lesion biopsy $(1 ; 1.4 \%)$, fibrin (1; 1.4\%), bronchoalveolar lavage $(1 ; 1.4 \%)$, leg lesion $(1 ; 1.4 \%)$, abdominal fluid $(1 ; 1.4 \%)$, catheter tip $(1 ; 1.4 \%)$, axillary secretion $(1 ; 1.4 \%)$, surgical wound fluid $(1 ; 1.4 \%)$, and tracheal secretion (1; $1.4 \%)$. Considering the patient gender and age, there was a prevalence of males $(50 ; 71.4 \%$ of 70 patients $)$ over- 60 -yearolds (37; 52.9\% of 70 patients), 21-59-year-olds (29; 41.4\%), and zero-20-year-olds $(4 ; 5.7 \%)$. The adult intensive care unit (ICU) was the hospital unit with the highest CRE isolation rate (19; $27.2 \%$ of 70 patients), followed by the general surgery unit $(18 ; 25.7 \%)$, the semi-intensive care unit $(8 ; 11.4 \%)$, adult emergency room $(8 ; 11.4 \%)$, the clinic $(6 ; 8.6 \%)$, nephrology unit $(3 ; 4.3 \%)$, treatment center for children and adolescents with cancer $(3 ; 4.3 \%)$, cardiac intensive care unit $(2 ; 2.9 \%)$, the maternal-infant and women's health unit $(1 ; 1.4 \%)$, psychiatric unit (1; $1.4 \%)$, and surgical block $(1 ; 1.4 \%)$. Antimicrobial susceptibility profiles of the isolates are shown in Table 1. Genotypic analysis based on PCR revealed the presence of the bla $a_{\mathrm{KPC}}$ gene in 56 isolates $(56 / 70 ; 80 \%)$, with the KPC activity also phenotypically demonstrated by the disk diffusion, $\mathrm{CN}$, and $\mathrm{BC}$ techniques (Table 2). The OXA ( $\left.b l a_{\mathrm{OXA}-48}\right)$ and MBL $\left(b l a_{\mathrm{NDM}}\right.$ and $\left.b l a_{\mathrm{GES}}\right)$ genes were not detected. All phenotypic methods (disk diffusion, $\mathrm{CN}$ and $\mathrm{BC}$ ) detected the KPC enzyme with 100\% sensitivity and specificity.

Carbapenem-resistant gram-negative bacilli may exhibit low susceptibility to antimicrobials, mainly to $\beta$-lactam antibiotics, because the production of carbapenemases is associated with porin loss, overexpression of efflux pumps, and the production of another $\beta$-lactamase [e.g., extended-spectrum $\beta$-lactamases $(\mathrm{ESBL})$ and AmpC $\beta$-lactamase $]^{8}$. This may explain why the $b l a_{\mathrm{KPC}}$ gene was not detected in 14 (out of 70) isolates in the present study. Similarly, Ribeiro et al., observed highlevel resistance against $\beta$-lactams and ciprofloxacin, and sensitivity to polymyxin B and amikacin in Enterobacteriaceae isolates in southern Brazil. In the same study, the $b l a_{\mathrm{KPC}}$ gene was identified in only 14 (14/345) isolates and, among the non-carbapenemase-producing isolates, ESBL and AmpC phenotypes were observed ${ }^{9}$. Differentiating a carbapenemaseproducing CRE from a non-carbapenemase-producing CRE is important. The selective pressure caused by indiscriminate use of broad-spectrum antibiotics in treating hospital infections may lead to resistance of endogenous bacteria (from the intestinal microbiota), but the reduction of susceptibility may also be associated with a rapid horizontal transmission (e.g., via healthcare workers, equipment contamination, and mobile genetic elements), contributing to the spread of heterogeneous resistance ${ }^{10}$.

In recent years, multiple resistance mechanisms have reduced the available therapeutic options, leading to an extensive use of aminoglycosides and polymyxins ${ }^{11}$. Although colistin showed good activity against CRE, 6(6/70) K. pneumoniae isolates were resistant to this antimicrobial agent $(\mathrm{MIC} \geq 16 \mu \mathrm{g} / \mathrm{mL})$. Santos et al. described a similar resistance profile of colistin-resistant Pseudomonas aeruginosa (9/231) isolated at the same hospital (HUSM) as in the present study ${ }^{12}$. This phenotype may be due to the lipopolysaccharides changes, such as modifications in lipid A by the addition of 4-amino-4-deoxy-L-arabinose (L-Ara4N) 
TABLE 2

Phenotypic and molecular evaluation of the isolated clinical specimens.

\begin{tabular}{|c|c|c|c|c|c|c|c|c|}
\hline \multirow{2}{*}{ Microorganism } & \multirow{2}{*}{ Unit } & \multirow{2}{*}{ Specimen } & \multirow{2}{*}{$n$} & \multicolumn{4}{|c|}{ Phenotype } & \multirow{2}{*}{$\begin{array}{c}\text { Genotype } \\
\text { PCR }\end{array}$} \\
\hline & & & & PBA & EDTA & $\mathrm{CN}$ & BC & \\
\hline Citrobacter freundii & Clinic & Urine & 1 & + & - & + & + & $b l a_{\mathrm{KPC}}$ \\
\hline Enterobacter cloacae & Adult emergency room & Urine & 1 & - & - & - & - & - \\
\hline \multirow[t]{17}{*}{ Escherichia coli } & Semi-intensive care unit & Rectal swab & 1 & + & - & + & + & $b l a_{\mathrm{KPC}}$ \\
\hline & \multirow{4}{*}{ Clinic } & SLB & 1 & + & - & + & + & $b l a_{\mathrm{KPC}}$ \\
\hline & & Feces & 1 & - & - & - & - & - \\
\hline & & Rectal swab & 1 & - & - & - & - & - \\
\hline & & Urine & 1 & + & - & + & + & $b l a_{\mathrm{KPC}}$ \\
\hline & Surgical block & Fibrin & 1 & + & - & + & + & $b l a_{\mathrm{KPC}}$ \\
\hline & \multirow[b]{2}{*}{ TCCAC } & Axillary secretion & 1 & + & - & + & + & $b l a_{\mathrm{KPC}}$ \\
\hline & & Rectal swab & 1 & + & - & + & + & $b l a_{\mathrm{KPC}}$ \\
\hline & \multirow{3}{*}{ Adult emergency room } & Blood & 1 & + & - & + & + & $b l a_{\mathrm{KPC}}$ \\
\hline & & \multirow{2}{*}{ Urine } & 3 & + & - & + & + & $b l a_{\mathrm{KPC}}$ \\
\hline & & & 2 & - & - & - & - & - \\
\hline & Psychiatric & Urine & 1 & - & - & - & - & - \\
\hline & \multirow{5}{*}{ Semi-intensive care unit } & Sputum & 1 & + & - & + & + & $b l a_{\mathrm{KPC}}$ \\
\hline & & Feces & 1 & + & - & + & + & $b l a_{\mathrm{KPC}}$ \\
\hline & & \multirow{2}{*}{ Rectal swab } & 1 & + & - & + & + & $b l a_{\mathrm{KPC}}$ \\
\hline & & & 3 & + & - & + & + & $b l a_{\mathrm{KPC}}$ \\
\hline & & Urine & 1 & + & - & + & + & $b l a_{\mathrm{KPC}}$ \\
\hline \multirow[t]{15}{*}{ Klebsiella pneumoniae } & MIWHU & Feces & 1 & - & - & - & - & - \\
\hline & \multirow{3}{*}{ General surgery unit } & Rectal swab & 3 & + & - & + & + & $b l a_{\mathrm{KPC}}$ \\
\hline & & \multirow{2}{*}{ Urine } & 3 & + & - & + & + & $b l a_{\mathrm{KPC}}$ \\
\hline & & & 1 & - & - & - & - & - \\
\hline & \multirow{2}{*}{$\begin{array}{c}\text { Cardiac intensive care } \\
\text { unit }\end{array}$} & Rectal swab & 1 & + & - & + & + & $b l a_{\mathrm{KPC}}$ \\
\hline & & Rectal swab & 1 & + & - & + & + & $b l a_{\mathrm{KPC}}$ \\
\hline & & Leg lesion & 1 & + & - & + & + & $b l a_{\mathrm{KPC}}$ \\
\hline & & Abdominal fluid & 1 & + & - & + & + & $b l a_{\mathrm{KPC}}$ \\
\hline & & Tracheal secretion & 1 & + & - & + & + & $b l a_{\mathrm{KPC}}$ \\
\hline & & & 7 & + & - & + & + & $b l a_{\mathrm{KPC}}$ \\
\hline & Intensive care unit & Rectal swab & 2 & + & - & + & + & $b l a_{\mathrm{KPC}}$ \\
\hline & & & 1 & - & - & - & - & - \\
\hline & & & 2 & + & - & + & + & $b l a_{\mathrm{KPC}}$ \\
\hline & & Urine & 2 & + & - & + & + & $b l a_{\mathrm{KPC}}$ \\
\hline & & & 2 & - & - & - & - & - \\
\hline
\end{tabular}

$\boldsymbol{n}$ : number of clinical specimens; PBA: phenylboronic acid; EDTA: ethylenediaminetetraacetic acid; CN: carba NP; BC: blue-carba; PCR: polymerase chain reaction; SLB: sacral lesion biopsy; TCCAC: treatment center for children and adolescents with cancer; MIWHU: maternal-infant and women's health unit; BAL: bronchoalveolar lavage; SWF: surgical wound fluid; +: positive; -: negative. 
and phosphoethanolamine (pEtN), and $m g r B$ gene mutations, responsible for the regulation of the PmrAB and PhoPQ twocomponent systems, as well as through plasmid-mediated mcr-1 gene, responsible for horizontal transfer of colistin resistance, necessitating monitoring of colistin use during therapy and reinforcing control practices during hospital infections to avoid the dissemination of resistance and loss of efficacy of this drug ${ }^{13}$.

In the present study, all phenotypic methods (disk diffusion, $\mathrm{CN}$ and $\mathrm{BC}$ ) detected the KPC enzyme activity with $100 \%$ sensitivity and specificity. Similarly, Giske et al. reported $100 \%$ sensitivity and $98 \%$ specificity of an assay that relied on the 3-aminophenylboronic acid for the detection of KPC in K. pneumoniae strains ${ }^{14}$. Further, García-Fernández et al. reported $100 \%$ specificity of both methodologies, with $100 \%$ and $98 \%$ sensitivity of $\mathrm{CN}$ and $\mathrm{BC}$, respectively ${ }^{15}$.

As demonstrated, the disk diffusion (PBA and EDTA) method, and $\mathrm{CN}$ and $\mathrm{BC}$ tests are characterized by high sensitivity and specificity, similarly to PCR. They might be incorporated into the workflow of a clinical microbiology laboratory, as they are quick, practical, and low-cost.

Simultaneous use of two or more of these methods increases the reliability of CRE detection and, together with the antimicrobial susceptibility profile data, they may inform the adequate treatment, assisting in the reduction of mortality and dissemination of resistance. The phenotype found in the Institution is worrying and the adoption of nosocomial infection control and prevent measures are necessary.

This research presented limitations of the study in terms of non-detection of other carbapenemases enzymes (OXA, MBL), which could interfere with sensitivity and specificity. Posteriorly, mechanisms such as efflux pump and porin loss will be evaluated, as well as molecular typability for outbreak analysis.

\section{Ethical considerations}

This research was approved by the Ethical Research Committee, Universidade Federal de Santa Maria, under approval number 38850614.4.0000.5346.

\section{Acknowledgments}

The authors would like to thank the Laboratório de Biologia Molecular of the Hospital Universitário de Santa Maria for the help provided in the performance of molecular methods.

\section{Conflict of interest}

The authors declare that there is no conflict of interest.

\section{REFERENCES}

1. World Health Organization (WHO). Antimicrobial resistance: global report on surveillance 2014. Geneva, Switzerland: WHO; 2014. 257 p.
2. Pitout JDD, Nordmann P, Poirel L. Carbapenemase-producing Klebsiella pnemoniae, a key pathogen set for global nosocomial dominance. Antimicrob Agents Chemother. 2015;59(10):5873-84.

3. Nordmann P, Gniadkowski M, Giske CG, Poirel L, Woodford N, Miriagou V, et al. Identification and screening of carbapenemaseproducing Enterobacteriaceae. Clin Microbiol Infect. 2012;18(5):432-8.

4. Agência Nacional de Vigilância Sanitária (ANVISA). Nota Técnica No 01/2013: Medidas de prevenção e controle de infecções por Enterobactérias multiresistentes. Brasília: ANVISA; 2013. 22 p.

5. Clinical and Laboratory Standards Institute (CLSI). Performance Standards for Antimicrobial Susceptibility Testing; Twenty-Fifth Informational Supplement. Vol 35, $\mathrm{n}^{\mathrm{o}}$ 3. M100-S25. Wayne, PA: CLSI, 2015. $240 \mathrm{p}$.

6. García-Fernándes S, Morosini MI, Gijón D, Beatobe L, RuizGarbajosa P, Domínguez L, et al. Detection of Carbapenemase Production in a collection of Enterobacteriaceae with characterized resistance mechanisms from clinical and environmental origins by use of both Carba NP and Blue-Carba tests. J Clin Microbiol. 2016;54(2):464-6.

7. Monteiro J, Widen RH, Pignatari AC, Kubasek C, Silbert S. Rapid detection of carbapenemase gene by multiplex real-time PCR. J Antimicrob Chemother. 2012;67(4):906-9.

8. Tsai YK, Liou CH, Fung CP, Lin JC, Siu LK. Single or in combination antimicrobial resistance mechanisms of Klebsiella pneumoniae contribute to varied susceptibility to different carbapenems. PLoS One. 2013;8(11):e79640.

9. Ribeiro VB, Andrade LN, Linhares AR, Barin J, Darini AL, Zavascki AP, et al. Molecular characterization of Klebsiella pneumoniae carbapenemase-producing isolates in southern Brazil. J Med Microbiol. 2013;62(11):1721-7.

10. Goodman KE, Simner PJ, Tamma PD, Milstone AM. Infection control implications of heterogeneous resistance mechanisms in carbapenem-resistant Enterobacteriaceae (CRE). Expert Rev Anti Infect Ther. 2016;14(1):95-108.

11. Rafailidis PI, Falagas ME. Options for treating carbapenem-resistant Enterobacteriaceae. Curr Opin Infect Dis. 2014;27(6):479-83.

12. Santos SO, La Rocca SM, Hörner R. Colistin resistance in nonfermenting Gram-negative bacilli in a university hospital. Braz J Infect Dis. 2016;20(6):649-50.

13. Poirel L, Jayol A, Nordmann P. Polymyxins: antibacterial activity, susceptibility testing, and resistance mechanisms encoded by plasmids or chromosomes. Clin Microbiol Rev. 2017;30:557-96.

14. Giske CG, Gezelius L, Samuelsen O, Warner M, Sundsfjord A, Woodford N. A sensitive and specific phenotypic assay for detection of metallo- $\beta$-lactamases and KPC in Klebsiella pneumoniae with the use of meropenem disks supplemented with aminophenylboronic acid, dipicolinic acid and cloxacillin. Clin Microbiol Infect. 2011;17(4):552-6.

15. García-Fernández S, Morosini MI, Gijón D, Beatobe L, RuizGarbajosa P, Domínguez L, et al. Detection of carbapenemase production in a collection of Enterobacteriaceae with characterized resistance mechanisms from clinical and environmental origins by use of both Carba NP and Blue-Carba tests. J Clin Microbiol. 2016;54(2):464-6. 